\title{
LA GESTIÓN DE LAS PERSONAS: LAS NUEVAS HABILIDADES QUE DEBEREMOS DESARROLLAR EN LAS PyMES, POST PANDEMIA
}

\author{
Amigo, Adriana \\ Bergmann, Cristian \\ Daffunchio, Adriana \\ Ortiz, Gisela
}

\section{Resumen:}

Gestionar personas, no es gestionar tan solo una categoría de recursos, es gestionar sentimientos, sensaciones, frustraciones, miedos, esperanzas, negaciones, ilusiones. Es gestionar sentimientos. Qué pasará con esas sensaciones una vez que todo esto haya pasado? No lo sabemos, sin embargo, debemos estar preparados para encontrarnos con "otras" personas, personas a las que les pasaron cosas, esas cosas que pasaron quizás nos enfrenten con una nueva manera de hacer, de rendir, de evaluar. Interpretar esas nuevas maneras de conjugar objetivos y recursos en pos de un resultado, quizás sea el camino por el que deberá transitar la nueva cultura empresarial de las pequeñas y medianas empresa, que representan hoy el sector de la economía, más afectado por esta pandemia decretada por la OMS, Covid 19.

Palabras claves: Gestionar - Recursos - Personas - Sensaciones - Camino - Cultura Empresarial

\begin{abstract}
:
Managing people, is not just managing a category of resources, it is managing feelings, sensations, frustrations, fears, hopes, denials, illusions. It is managing feelings. What will happen, to those sensations, once all this has happened? We do not know, however, we must be prepared to meet "other" people, people to whom things happened, those things that happened, may confront us with a new way of doing, of performing, of evaluating, Interpreting these new ways of combining objectives and resources in pursuit of a result, may by the path that the new business culture of small and mediums-sized companies, will have to travel, which today represent the sector of the economy, most affected by this decreed pandemic by WHO Covid 19.
\end{abstract}

Keywords: Managing - Resources - People - Sensations - Way - Business - Culture 


\section{INTRODUCCIÓN}

Los nuevos escenarios que platea la pandemia: la incertidumbre, la adaptabilidad, el compromiso social, los escenarios de crisis, nos plantean nuevos requerimientos de las personas al conformar los equipos de trabajo. ¿Qué será más importante la actitud o la aptitud? En cuyo caso ¿Cómo será la función del nuevo líder?. ¿Deberán cambiar los líderes, o tan solo los seguidores?

Nos proponemos, adentrarnos en este camino investigativo, sobre estas reflexiones, que son nuestros interrogantes no resueltos, dado que la nueva realidad, nos plantea tan sólo el horizonte del corto plazo, tan desafiante aún en el corto horizonte en el que nos impone profundos y rápidos cambios. No podemos no estar atentos, es una oportunidad única para re pensar el modelo empresa y para aportar una propuesta de mejora al desarrollo de capacidades únicas, irrepetibles, de difícil imitación, valoradas por el mercado y de difícil venta. (Amigo, 2017)

\section{LA GESTIÓN DE PERSONAS: ¿PROCESO, MÉTODO O HERRAMIENTA?}

El liderazgo adquiere un rol central en las organizaciones, especialmente en un contexto de alta imprevisibilidad e incertidumbre como el actual, en el cual no están todavía claras las nuevas leyes o normas preponderantes en la llamada "nueva normalidad".

Esto implica un enorme desafío en la gestión de las personas, especialmente en las PyMes.

Ahora bien, cuando nos referimos a la gestión de las personas, es dable plantear si nos referimos a un proceso, un método o una herramienta de gestión.

Según los autores Gómez, Balkin, Cardy (2008), el entorno tiene una gran influencia sobre la gestión de las personas, puesto que se caracteriza por cambios vinculados a la globalización, internacionalización e integración de las empresas, sumado a la evolución del trabajo, la diversidad y el exponencial crecimiento de las tecnologías de la información y la comunicación.

La necesidad de las empresas de adaptarse para competir, de adaptarse para construir el futuro, de adaptarse para sobrevivir en entornos de crisis, implica que éstas puedan realizar el ajuste necesario en la gestión de las personas.

He aquí la importancia de las capacidades, las cuales reflejan, según Chiavenato (2007), la sinergia entre los recursos con los que cuenta la organización, lo que posibilita su aplicación en la construcción de una ventaja competitiva. Las capacidades son conjuntos complejos de habilidades y aprendizaje colectivo, el cual asegura la ejecución de 
actividades funcionales mediante procesos organizacionales. La administración del conocimiento es la actividad determinante de la organización para lograr esa ventaja competitiva.

Entre los recursos, podemos destacar los activos físicos, financieros, tecnológicos, humanos e imagen corporativa.

Pero el análisis quedaría incompleto si no concebimos que una correcta conjunción de estos genere capacidades estratégicas, funcionales y operacionales.

Para Hamel y Prahalad (1994; 1999), la arquitectura organizacional necesita proporcionar coherencia en la ubicación de recursos y en el desarrollo de una estructura administrativa adecuada a esa ubicación a fin de crear una cultura administrativa dinámica, trabajo en equipo, capacidad de cambio y disponibilidad para compartir recursos que permitan proteger las habilidades singulares y para pensar el largo plazo.

De allí se desprende que esa correcta "ecualización" de recursos da como resultado una de las principales fuentes de competitividad de las empresas.

Pero los autores, profundizan en un concepto que permite a las organizaciones trascender y competir por el futuro. La "arquitectura estratégica" podrá construirse si los directores tienen claro cuáles son los nuevos beneficios o funcionalidades que se ofrecerán a los clientes en la próxima década, cuáles son las competencias esenciales que se necesitarán para ofrecer esos beneficios y sobre cómo habrá de modificarse la relación con los clientes para que éstos puedan acceder más eficazmente a esos beneficios.

En esencia, la "arquitectura estratégica" permite analizar la utilización de nuevas funcionalidades, la adquisición de nuevas competencias o la migración de competencias existentes y la reconfiguración de la relación con los clientes.

En los tiempos actuales y en la post-pandemia, toma notoria relevancia lo planteado por los autores en términos de como apalancar los recursos, para lo cual se describen cinco maneras fundamentales: concentrándolos, acumulándolos, complementando, conservando o recuperando los recursos.

Si entendemos a la gestión de las personas como un proceso, tal como lo define Chiavenato (2008), se establecen cinco procesos básicos: integrar, organizar, retener, desarrollar y auditar a las personas, los cuales están interrelacionados y son interdependientes.

Continuando con el planteo del autor, podríamos pensar que la gestión de las personas es una política, a partir de lo cual podemos entenderla como la manera en que las 
organizaciones desean tratar a sus miembros para lograr por medio de ellos los objetivos organizacionales, al proporcionar condiciones para el logro de los objetivos individuales.

Desde este enfoque de la gestión de las personas como proceso o política, perdemos de vista el enorme aporte de las personas a la estrategia organizacional. Si pensamos que las estrategias -cualesquiera de ellas- son personas interactuando en un marco que facilita y promueve la construcción de valor para los públicos de interés, la caracterización como sólo un elemento táctico u operacional, luce incompleta a los fines propuestos en el presente artículo.

Aquí se desprende un elemento que podría funcionar como catalizador del cambio y de la adaptabilidad de las organizaciones a una "nueva normalidad".

Volviendo a uno de los planteos principales del presente artículo, ¿cómo será la función del nuevo líder? ¿Deberá adaptarse sólo el líder o también sus seguidores?

Según un artículo publicado en el Diario La Nación el 10 de agosto del 2020, los autores Marchiori y Hatum plantean interesantes interrogantes sobre el liderazgo político mundial: ¿Qué se puede aprender de los líderes de esos países que -como Alemania- han demostrado mayor capacidad de capear el temporal? ¿Qué hicieron? ¿Qué pasos siguieron? Por el contrario, ¿qué aprendizajes están dejando los países más afectados? En un análisis de los patrones de comportamiento, los autores destacan en primer lugar la capacidad de realizar el diagnóstico de la situación, es decir la aptitud de reconocer que se está frente a un problema y asumirlo. El ejemplo de Ángela Merkel en Alemania nos permite ver un liderazgo centrado en el problema y en las soluciones, al contrario de lo sucedido en Inglaterra con Boris Johnson, quien inicialmente tomó medidas gubernamentales evasivas hasta tener que enfrentarse con la enfermedad en carne propia.

En segundo lugar, generar una visión, implica canalizar los esfuerzos de los seguidores detrás de un camino, generando compromiso colectivo.

Posteriormente, resulta crucial trazar un plan de acción que ayuda a reducir la incertidumbre de la población o del equipo de trabajo.

La comunicación, adquiere un rol central en brindar información que de tranquilidad, con seriedad y basado en datos empíricos contrastados científicamente.

Finalmente, el control y reajuste de las políticas y acciones implementadas permite verificar el cumplimiento de los objetivos trazados, analizar las causas de los desvíos y tomar las medidas correctivas necesarias. 
En suma, el desarrollo de las características explicadas para los líderes estatales resulta igual de importante en el liderazgo de empresas Pymes, a quienes la crisis ha puesto en una situación de gran stress financiero, comercial y operacional.

La post pandemia será una inmejorable oportunidad de desarrollar nuevas habilidades. Según se sostiene en el informe "Resiliencia humana: las personas y sus necesidades actuales" elaborado por Accenture Research en base a un estudio basado en una encuesta a más de 15.600 empleados en 10 países de 15 sectores económicos, los colaboradores en las empresas trasladaron dos necesidades fundamentales que preceden a todas las demás: por un lado, un equipo directivo centrado en la compasión y el cuidado de sus empleados y la confianza en la gestión de la empresa ante el futuro.

En el estudio, los empleados pidieron por permisos para trabajar de una manera distinta para ser más productivos y cuidar de la salud mental, así como recibir información periódica para poder realizar el trabajo y responder con resiliencia.

En la actualidad, muchas personas se incorporan a una compañía porque se identifican con sus valores o propósito. En tiempos de crisis, esos valores y ese propósito deben destacarse más que nunca. ¿Por qué? Porque son la base del sentido de conexión y pertenencia de los empleados.

Según el artículo "Creatividad y resiliencia": las habilidades más valoradas para afrontar la "nueva normalidad", entre las nuevas habilidades que será necesario desarrollar para la nueva normalidad, se destacan la creatividad, la gestión del cambio y la capacidad de resiliencia, a las que se suman dos competencias blandas para los y las líderes PyMEs: empatía y responsabilidad.

La experiencia del Departamento de Recursos Humanos de banco HSBC en Argentina, permitió identificar 36 habilidades del futuro agrupadas en 9 comportamientos clave a desarrollar: curiosidad; creatividad; conectividad; resiliencia; engagement de clientes; desarrollo de otros; cambio y transformación; gestión de comunicaciones y mentalidad de crecimiento.

Esto implica que las empresas PyMEs, continuarán concentrando sus esfuerzos en el mantenimiento de los recursos que permitan sostener las capacidades distintivas, no obstante, deberán adaptar la gestión de las personas desarrollando nuevas aptitudes que permitirán dotar del sentido de trascendencia necesario para superar la coyuntura y continuar, tal como lo plantean Hamel y Prahalad, repensando el futuro. 


\section{LA TRANSVERSALIDAD DE LA GESTIÓN DE PERSONAS.}

Una estrategia de Recursos Humanos $(\mathrm{RRHH})$ refiere a la utilización por parte de la empresa, de los RRHH para mantener una ventaja frente a sus competidores en el mercado. Una táctica de $\mathrm{RRHH}$ es una política o un programa concreto que ayuda a avanzar hacia el objetivo estratégico de la empresa.

Gómez, Balkin y Cardy (2008), definen al "rápido cambio del entorno" como uno de los siete retos del entorno más importantes a los cuales se enfrentan las organizaciones hoy en día (los otros retos son: el crecimiento de Internet, la diversidad de los trabajadores, la globalización, la legislación, la evolución del trabajo y del papel de la familia, la carencia de cualificación y el crecimiento del sector servicios).

Con respecto a la rapidez de los cambios en el entorno, es fundamental la rápida adaptación para sobrevivir, y los $\mathrm{RRHH}$, en este sentido, siempre resultan clave en un sistema de respuesta eficaz. La llegada de la pandemia del COVID 19 represento para todas las organizaciones un desafío sin precedentes, y un cambio en el entorno al cual debieron hacer frente en muy poco tiempo.

Según Gómez, L., Balkin, D., y Cardy, R. (2008), algunas de las medidas adoptadas por las empresas cuando se enfrentan a una creciente presión por ser más productivas y trabajar con ciclos de vida del producto cada vez más cortos, tienen que ver con reducir el estrés de los trabajadores. En este sentido, las empresas deben incorporar servicios o beneficios que incrementen el bienestar y la comodidad en el entorno laboral.

La formación del personal será otro punto fundamental a atender. La formación se centra habitualmente en ofrecer a los empleados habilidades concretas o en ayudarles a corregir deficiencias en su rendimiento, y se diferencia del desarrollo del personal pues este último trata de un esfuerzo que consiste en ofrecer a los trabajadores las habilidades que la organización necesitará en el futuro. (Gómez et al., 2008). En un contexto de crisis y cambio como el actual la formación será clave para reducir esa brecha de conocimiento existente. "Las competencias que el personal necesita y hoy no posee constituyen la "brecha de aprendizaje", es decir, lo que necesitan aprender para mejorar su desempeño." (Amigo, Blanque, Latorre y Yanda, 2013, p.4).

Es importante destacar que en las prácticas habituales de R.R.H.H., el criterio para agrupar pericias es el puesto de trabajo. Sin embargo estas pericias que ayer parecían adecuadas para un determinado puesto, en el contexto actual, ya no parecen serlo. "La dinámica del mundo y de las organizaciones en particular, nos está mostrando que el puesto de trabajo puede no ser el mejor "paraguas" para agrupar habilidades" (Gore y 
Mazzini, 1998, p.3). Para estos autores, el concepto de "competencias" que designa modelos mentales, conocimientos, o habilidades vinculadas al trabajo puede resultar más adecuado que el de puesto de trabajo, en tanto los puestos de trabajo cambian, se transforman o desaparecen y los conocimientos asociados a estos puestos se modifican y obsolecen.

Finalmente, el liderazgo será otra variable que deberá adaptarse a la nueva normalidad. Es un antes y un después de la pandemia, pues lo que hasta ayer eran zonas de confort, ahora son espacios de incertidumbre, a los cuales deben acoplarse todos las personas. El liderazgo trata ahora de corregir algunas cuestiones, deshacer otras y emprender nuevos caminos.

Las personas atraviesan las organizaciones de punta a punta, sin importar el rol que ocupen, y más aún en las pequeñas y medianas empresas, por la cercanía que estas generan. En una crisis como la actual, será necesario un liderazgo que pueda gestionar los recursos humanos de modo tal que estos puedan adaptarse rápidamente a los nuevos requerimientos del entorno. El líder en este nuevo orden deberá lograr que las competencias individuales generen valor y se integren a las competencias centrales de la organización, a fin de aprovechar las oportunidades que el nuevo entorno presente.

Las pequeñas y medianas empresas, pueden aprovechar estos cambios del entorno y transformarlos en oportunidades, ya que pueden adaptarse mejor y más rápido en relación a las organizaciones más grandes y burocráticas. Sin embargo, para que las Pymes estén en condiciones de aprovechar estas oportunidades, necesitarán disponer de los recursos adecuados y hacer un buen uso de los mismos (Fernandez y Revilla, 2008).

Fernández y Revilla (2008) observan asimismo una situación ambivalente en lo que refiere específicamente a los recursos humanos en las PyMEs. Por un lado se puede esperar personal motivado y con una fuerte vinculación con su empresa, mayor flexibilidad, cercanía entre dirección y empleados, mayores flujos de información y más participación, que posibilitan que los trabajadores adquieran conocimientos tácitos de la empresa. Por el contrario, observan niveles retributivos inferiores a los de las grandes empresas al igual que menores posibilidades de promoción y desarrollo profesional, lo cual dificulta la contratación de personal cualificado, tanto técnico como de gestión.

En este sentido, a fin de hacer frente a los desafíos y las oportunidades que plantea el entorno, resulta clave para las pymes superar estas limitaciones así como fortalecer y capitalizar aquellos puntos positivos con que cuentan. 
Sera fundamental entonces, la gestión de los recursos adecuadamente a fin de desarrollar capacidades que permitan que la empresa haga algo mejor que las demás pese a no disponer de ningún recurso privilegiado (Fernández y Revilla, 2008).

Dicho esto, debe tenerse en cuenta que cualquier recurso o capacidad no es una fuente de ventaja sostenible frente a la competencia. Los atributos que se requieren de un recurso para lograr la sostenibilidad son: unicidad, inimitabilidad, durabilidad, e insustituibilidad (Munuera Alemán y Rodriguez Escudero, 2007).

En este sentido, los recursos humanos pueden considerarse como fuentes de ventajas competitivas sostenibles claves, especialmente por su cualidad de no poder imitarse, pues cada individuo es único. De ahí su importancia dentro de la estrategia comercial integrada de la empresa. Su gestión por tanto debe atravesar a toda la organización, y no estancarse en un solo departamento o área. Los directivos por lo tanto con la ayuda del departamento de Recursos Humanos deben implantar las estrategias de RRHH que hayan elegido. "Las competencias individuales generan valor en tanto estén integradas a las competencias centrales de la empresa" (Gore y Mazzini, 1998, p.5).

\section{LAS HABILIDADES SOFT Y LAS HABILIDADES HARD.}

El año 2020, implicó un cambio violento en el modo de trabajo de las empresas y organizaciones de todo el mundo; y sin duda alguna se reflejó con mucha fuerza en el departamento de recursos humanos, que debió dirigir y poner en funcionamiento la metodología de teletrabajo con una velocidad inesperada y sin ninguna preparación previa. Si bien, es cierto que en otros lugares y empresas del mundo, el trabajo Home Office se venía imponiendo, en nuestro país las experiencias eran muy escasas, y el 20 de marzo 2020, la pandemia COVID-19 obligó a muchos trabajadores a permanecer en sus hogares y su forma de trabajo cambió radicalmente.

Durante los primeros días la tensión se hizo extrema a raíz de la urgencia en la toma de decisiones y la rapidez necesaria para ejecutar las mismas como por ejemplo, la compra de equipos informáticos para los empleados que estaban en sus casas, las conexiones de trabajo remoto, etc. Tras éste primer impacto, fue posible comenzar a analizar la efectividad de las tareas realizadas de éste modo, es decir, si realmente era posible llevar a cabo todas las tareas de los puestos que se enviaron a casa y lo más importante, si quienes desempeñaban dichos puestos contaban con los requerimientos necesarios para realizar teletrabajo. 
Como es conocido, el decreto 297 del PEN dispuso el aislamiento social, preventivo y obligatorio, con el fin de mitigar el impacto sanitario del COVID 19, lo cual produjo una disminución de trabajadores en sus lugares habituales motivo por el cual las empresas debieron recurrir al mercado laboral para cubrir puestos. Es allí cuando surgen cambios en la selección del personal, ya que habilidades o competencias soft que no eran requeridas para todos los puestos pasan a tener un papel preponderante, tal es el caso de el manejo de herramientas digitales, flexibilidad y proactividad y comunicación.

Según Spencer y Spencer (Alles, 2002, p. 7); competencia es una característica subyacente en un individuo que está causalmente relacionada a un standard de efectividad y/o una performance superior en un trabajo o situación. De allí que las competencias sean consideradas en todos los procesos relacionados con la vida o carrera laboral dentro de las empresas u organizaciones, es decir, selección, evaluación, promociones, acceso a beneficios y gestión de personas en general.

Las empresas están formadas por personas y de acuerdo a lo expresado por Claude Levy -Lebayer (Alles, 2002, p. 12), las competencias de las empresas están constituidas ante todo por la integración y la coordinación de las competencias individuales de su personal. De allí la importancia para la empresa de administrar bien su stock de competencias tanto actuales como potenciales.

El manejo de herramientas digitales incluye a todas las disponibles y utilizadas por la empresa que selecciona personal nuevo o temporario.

Martha Alles en su Diccionario por competencias, se refiere a Flexibilidad (Alles, 2002, p. 37), trata acerca de la capacidad para adaptarse rápidamente y trabajar con eficacia en distintas y variadas situaciones y con personas o grupos diversos. Implica apreciar perspectivas diferentes. Supone entender y valorar distintas posturas o puntos de vista encontrados, adaptando su propio enfoque a medida que la situación cambiante lo requiere y promoviendo los cambios en la propia organización o en las responsabilidades a su cargo.

Al evaluar a la persona que desempeñará el puesto será importante analizar si el candidato ve el cambio como una oportunidad y no como una amenaza y si mantiene un buen nivel de desempeño aún con cambios en las condiciones de realización de sus tareas. Ajusta sus acciones ante cambios en la planificación o lineamientos de la dirección.

Al definir la competencia Iniciativa o Proactividad (Alles, 2002, p. 39), se dice que es la capacidad para actuar anticipándose a los hechos, idear e implementar soluciones a 
nuevas problemáticas con decisión e independencia de criterio. Implica la habilidad para responder con rapidez, eficacia y eficiencia ante nuevos requerimientos. Es la predisposición a emprender acciones, crear oportunidades y mejorar resultados, sin necesidad de un requerimiento externo que lo impulse.

Al evaluar a la persona tanto que postula o que ocupa el puesto, deberá tenerse en cuenta si cumple sus tareas sin realizar continuas consultas, no requiere supervisión estrecha Es autónomo para proponer pautas, métodos o criterios de organización para su tarea Actúa antes de que se lo pidan, busca información necesaria y resuelve los obstáculos que se le presentan .Reacciona rápidamente para evitar que un problema menor se convierta en grave. Muestra disposición a buscar nuevas tareas, desafíos y oportunidades.

La competencia Modalidad de Contacto es definida por la misma autora (Alles, 2002, p. 63) como: la capacidad de demostrar una sólida habilidad de comunicación. Asegura una comunicación clara. Alienta a otros a compartir información y valora las contribuciones de los demás.

Otra competencia que cobra relevancia es la Innovación (Alles, 2002, p. 71), entendida como la capacidad para modificar las cosas incluso partiendo de formas o situaciones no pensadas con anterioridad. Implica idear soluciones ante problemas o situaciones requeridas por el propio puesto, la organización, los clientes o el segmento económico donde actúa. La realidad actual podría sumarse claramente a ésta lista propuesta por Martha Alles.

Es muy probable que estas definiciones de habilidades y sus descriptores, ya se encuentren en los diccionarios de competencias que muchas empresas poseen o en los Manuales de evaluación de puestos, pero lo novedoso será ajustarlos a puestos que quizás antes del Covid-19 no lo requerían.

En éste marco toma relevancia el proceso de onboarding planificado por las empresas. Cabe destacar que la implementación este tipo de programas se torna imprescindible para las PyMES, ya que no es necesario realizar una erogación económica demasiado alta y disminuye los costos laborales derivados del pago de salarios sin retorno y de los recurrentes gastos en selección (Santillana, 2020).

Las PyMEs que quieran implementar procesos onboarding podrán hacerlo por medio de videos o cursos de e-learning o de plataformas virtuales para nuevos empleados. Estas herramientas son de bajo costo y ya han sido probadas en países como España, donde 
por medio de éste proceso las empresas han tratado de formar, informar y motivar a sus empleados, tal es el caso de la empresa INDRA.

En definitiva este es un desafío más, que deberán enfrentar las empresas en su búsqueda de nuevas estrategias de negocio para la generación de valor en una sociedad que seguramente habrá cambiado.

\section{CONCLUSIONES}

- Los cambios manifestados, en tiempos contemporáneos, atravesados por la declaración de pandemia mundial decretada por la OMS, COVID 19, nos platean nuevos escenarios desafiantes y anclajes de las nuevas plataformas estratégicas para operar cambios estructurales en las organizaciones.

- Frente a la exigencia de la adaptabilidad, las empresas y especial las PyMEs, pueden tomar la oportunidad de cambiar bajo decreto, pues "el no cambio", representa "el no futuro".

- Cuando dos grandes autores como Hamel y Prahalad, nos invitan a repensar el futuro, si adecuamos ese título a nuestro presente, encontramos que nuestro futuro más que nunca depende de nuestro presente, por lo que el cómo y la decisión del "sí cambio" adecuado a una propuesta de valor comprometida, ética y empática con esta nueva realidad, están instalando un concepto siempre vigente cual es, el presente compromete y asegura el futuro de la organización.

- Las posibilidades pueden verse afectadas, según cómo las miremos, observemos, analicemos y apalanquemos, sólo que deberemos comenzar, replanteando los negocios, a partir de las nuevas realidades, en las que la carencia del recurso y la presencia de la dificultad, serán inspiradores de estrategias basadas en el valor, desde una visión compartida por todos quienes componemos los equipos de trabajo, los que quizás ya no tengan un solo líderes, será necesario convocar a los líderes formales y a los líderes informales, a los seguidores de los líderes, a los enterpreneur y a los enterpreneurships, pues tener éxito dejará de ser un objetivo, un objetivo tan solo será alcanzar el próximo objetivo... 


\section{REFERENCIAS BIBLIOGRÁFICAS}

Alles, M. (2002) Dirección estratégica de Recursos Humanos. Gestión por Competencias: el diccionario. Editorial Granica. Buenos Aires. Argentina

Amigo, Adriana. (2009): Negocios con Valor. Editorial Fundación Ross. $1^{\circ}$ Edición. Rosario. Argentina.

Amigo, Adriana. (2012): Negocios con Valor. Editorial Fundación Ross. $2^{\circ}$ Edición. Rosario. Argentina.

Amigo, Adriana. (2017): Negocios con Valor. Una metodología para generar ventajas competitivas sostenibles. Editorial Académica Española. $3^{\circ}$ Edición. $1^{\circ}$ Edición Europea. Madrid. España

Amigo, Adriana y otros (2013): Cómo generar una imagen de marca positiva desde la gestión del capital humano a través del desarrollo de competencias distintivas. XVIII Jornadas de Investigación de la Facultad de Ciencias Económicas y Estadística. Rosario, Argentina.

Carrión Maroto, Juan. (2006): Estrategia. De la Visión a la acción. ESIC. $2^{\circ}$ Edición. Madrid. España.

Chiavenato A. (2007): Administración de Recursos Humanos. McGraw Hill Interamericana. $5^{\circ}$ Edición. México.

Fernández, Z. y Revilla, A. (2008): Hacer de la necesidad virtud. Los recursos de las PyMES. Universidad Carlos III. Madrid. 2008.

Gómez, L., Balkin, D., y Cardy, R. (2008): Gestión de Recursos Humanos Pearson Educación SA $5^{\circ}$ Edición. Madrid. España.

Gore E. y Mazzini M. (1998): Desarrollo de competencias y aprendizaje organizacional. XI Congreso Nacional de Desarrollo y Capacitación. Asociación de Desarrollo y Capacitación de la Argentina. Mar del Plata, Argentina.

Hamel G., Prahalad C.K. (1994; 1999). Compitiendo por el futuro: estrategia crucial para crear los mercados del mañana. Ariel Sociedad Económica. Barcelona, España.

Munuera Alemán, J. y Rodríguez Escudero, A. (2007) Estrategias de marketing. Un enfoque basado en el proceso de dirección. ESIC. Madrid. España.

Publicaciones en webs: 
Marchiori E. A. y Hatum A. Liderazgos bajo la lupa. Qué pueden aprender los CEO del manejo de los presidentes. Diario La Nación. Extraído de https://www.lanacion.com.ar/economia/negocios/liderazgos-bajo-lupa-quepueden-aprender-ceo-nid2417370.

Samela G. Creatividad y resiliencia: las habilidades más valoradas para afrontar la "nueva normalidad". Diario Clarín. Extraído de https://www.clarin.com/economia/creatividad-cambio-resiliencia-habilidadesvaloradas-nueva-normalidad- 0 BgIR9A nl.html

Romero, I. (2020) El proceso onboarding, un aliado contra la fuga de talento Revista Capital Humano, 350 - Recuperado de http: https:// https://factorhuma.org/es/actualitat/noticias/14472-el-proceso-onboarding-unaliado-contra-la-fuga-de-talento 